2

4

5

6

\title{
Functional assessment and structural basis of antibody binding to human
}

\section{papillomavirus capsid}

Xiao Zhang, ${ }^{\text {a, b }}$, Shaowei Li ${ }^{\text {a, b, c }}$, Yorgo Modis ${ }^{\text {, }}$, Zhihai Li ${ }^{\text {a, c }}$, Jun Zhang ${ }^{\text {a, b }}$, Ningshao $\mathrm{Xia}^{\mathrm{a}, \mathrm{b}, \mathrm{c} *}$, Qinjian Zhao ${ }^{\mathrm{a}, \mathrm{b}} *$

${ }^{a}$ State Key Laboratory of Molecular Vaccinology and Molecular Diagnostics,

National Institute of Diagnostics and Vaccine Development in Infectious Diseases,

Xiamen University, Xiamen, Fujian 361005, PR China

${ }^{\mathrm{b}}$ School of Public Health, Xiamen University, Xiamen, Fujian 361005, PR China

${ }^{\mathrm{c}}$ School of Life Science, Xiamen University, Xiamen, Fujian 361005, PR China

${ }^{\mathrm{d}}$ Department of Medicine, University of Cambridge, MRC Laboratory of Molecular

Biology, Francis Crick Avenue, Cambridge, CB2 0QH, United Kingdom

${ }^{*}$ Corresponding author: Qinjian Zhao, School of Public Health, Xiamen University, Xiamen, Fujian 361005, PR China, Phone: +86-592-2180936, Fax: +86-592-2181258, qinjian_zhao@xmu.edu.cn, or Ningshao Xia, nsxia@xmu.edu.cn.

Running Head: Functional epitopes on human papillomavirus capsid

Keywords: human papillomavirus, pseudovirions, neutralizing antibodies, functional epitope, flexible loops, viral capsid 
HPV, human papillomavirus; BPV, bovine papillomavirus; VLP, virus-like particle; $\mathrm{Ab}$, antibody; Ag, antigen; PsV, pseudovirion; HSPGs, heparin sulfate proteoglycans; alkaline phosphatase; cLIA, competitive Luminex immunoassay. 


\section{Summary}

Persistent high-risk human papillomavirus (HPV) infection is linked to cervical cancer. Two prophylactic virus-like particle (VLP)-based vaccines have been marketed globally. Here, we review the approaches employed to generate HPV pseudovirions (PsV) that mimic native virions and to assess the neutralization activity of HPV antibodies in patient sera. The PsV-based neutralization assay was developed to study the virology of HPV and to evaluate vaccine efficacy. Specifically, this system has been used to evaluate the efficacy of neutralization antibodies in sera elicited by vaccination or natural infection or to assess the functional characteristics of monoclonal antibodies. We also review the antibody binding modes observed in virus-antibody complexes from the work done on virions, PsVs or VLPs. The neutralizing epitopes are localized on surface loops of the L1 capsid protein, at various locations on the capsomere. Different neutralization antibodies exert their neutralizing function via different mechanisms. Some antibodies neutralize the virion by inducing conformational changes in the viral capsid, which can result in concealing the binding site for a cellular receptor like $1 \mathrm{~A} 1 \mathrm{D}-2$ against dengue virus, or inducing premature genome release like E18 against EV71 [1, 2]. Higher-resolution details on the epitope composition of HPV neutralizing antibodies would shed light on the structural basis of the highly efficacious vaccines and aid the design of next generation vaccines. For the current vaccines, certain assays can be developed on the basis of epitope information for jmproved quality analysis and for

Yorgo Modis 11/10/2015 12:04 PM

Deleted: neutralizing Yorgo Modis 11/10/2015 12:04 PM

Deleted: exert their neutralization functions Yorgo Modis 11/10/2015 12:07 PM

Deleted: the epitopes recognized by Yorgo Modis 11/10/2015 12:07 PM Deleted: Yorgo Modis 11/10/2015 12:07 PM

Deleted: the Yorgo Modis 11/10/2015 12:06 PM

Deleted: as

Administrator 11/3/2015 9:51 AM

Deleted: Some neutralizing antibodies induce conformational changes in the viral capsid, suggesting that the virus may recognize multiple cellular receptors during cell entry. Yorgo Modis 11/10/2015 12:09 PM Deleted: better 
the comparability exercise for process improvement or process scale up to meet market demand. For next generation vaccine design, better understanding of the structural basis of the type-specific functional epitopes could aid the design of antigens with cross-type protection activity through epitope redesign or recombination such as loop grafting in hybrid VLPs [3-5].
Yorgo Modis 11/10/2015 12:09 PM

Deleted: - 


\section{Introduction}

Human papillomavirus (HPV) is recognized as the causative agent of cervical cancer and virtually all cervical cancers are related to the presence of oncogenic HPV DNAs [6-8]. In addition, genital warts are also caused by non-oncogenic HPV types although they are regarded as benign. HPVs are small non-enveloped DNA viruses that belong to the Papovaviridae family. Two late viral proteins, the major L1 and the minor $\mathrm{L} 2$, form the viral capsid $[9,10]$. The $\mathrm{L} 1$ protein can spontaneously self-assemble into virus-like particles (VLPs) [11]. Currently, two globally licensed VLP-based vaccines, Gardasil ${ }^{\circledR}$ and Cervarix $^{\circledR}$ (initially introduced in 2006) have been proven to effectively prevent HPV infection and to reduce HPV-related morbidity [12-17]. The two vaccines can induce a strong immune response and elicit functional L1-specific antibodies. The conferred protective immunity is primarily due to the viral neutralization function of the antibodies that may bind the incoming authentic virions[18-21]. As reported in the literature, all the identified antibodies against L2 such as RG1, 14H6, K4L2 andK18L2 were raised using the recombinant

\section{L2 protein. The neutralizing activities of these antibodies were demonstrated with the}

PsV-based neutralization system.[22-24], Because viral neutralization appears to involve both L1 and L2, the pseudotyped virus (PsV) neutralization model was developed to study the virology of HPV and evaluate the vaccine potential of the VLPs, The PsV-based neutralization assay was utilized to evaluate the efficacy of neutralization antibodies in sera elicited by vaccination or natural infection. Different PsV systems encapsidating the SEAP or RFP as the reporter have been used to

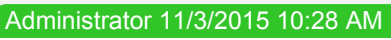

Deleted:

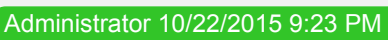

Deleted: and mortality

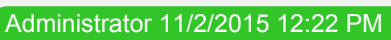

Deleted:

Yorgo Modis 11/9/2015 2:58 PM

Deleted: by

Yorgo Modis 11/9/2015 2:58 PM

Deleted: $\mathrm{s}$

Yorgo Modis 11/9/2015 2:58 PM

Deleted: actually

Yorgo Modis 11/9/2015 2:58 PM

Deleted: y

Administrator 11/2/2015 9:03 PM

Deleted: In a natural infection, antibodies

against the minor L2 protein are also able to neutralize HPV. 
evaluate the neutralization titers in the serum obtained from VLP-vaccinated women, or women naturally infected with HPV, or prenatal pregnant women [25-27]. PsV was

generated by transfecting the L1 and L2 genes into the 239FT cells. PsVs encapsidating the reporter gene were harvested by lysing the cells. Subsequently, the antibodies with a serial dilution was mixed with the titered PsV and then the mixture was added into the 239TT cells. Antibody-mediated PsV neutralization is evaluated by detecting the reporter activity such as SEAP activity [28]. Neutralizing antibodies play a key role in preventing viral infection via different mechanisms [29]. The neutralization mechanisms were reflected in the different binding sites located on the major capsid protein L1 or the minor capsid protein L2. If the high-resolution structures of $\mathrm{Ab}-\mathrm{Ag}$ complexes were determined, a better understanding of the neutralization mechanism of different mAbs would be achieved. However, this information is rather limited at this stage. Such knowledge of the details of identification and characterization of binding sites for neutralization antibodies would aid in the design of the next generation of vaccines and in the quality control of existing vaccines during vaccine manufacturing [30].

\section{Mimicking native virions with HPV pseudovirions}

The lack of permissive and productive cell cultures for HPV has impeded the study of virus-neutralizing mechanisms of antibodies, the monitoring of viral infection and other virological studies [31]. With the need for measuring neutralizing antibodies to high-risk genital HPVs such as HPV16 and HPV18 in the evaluation of vaccine 
efficacy, pseudovirion expression systems have been developed for the in vitro generation of HPV pseudovirions and for a quantitative in vitro assay for infectivity evaluation (Figure 1).

In early studies, Zhou et al. used recombinant vaccinia virus to produce bovine papillomavirus type-1 (BPV-1) VLPs containing both L1 and L2 capsid proteins in vitro. This system served as an in vitro model to identify the BPV-1 cellular receptor [32]. In a separate study, Semliki Forest virus was chosen by Roden et al. to express the papillomavirus virion proteins for generating pseudotyped papillomaviruses in vitro [33]. This system has not only permitted the analysis of the ability of antibody cross-type neutralization but also provided insight into the mechanism and specificity of papillomavirus genome packaging and infection.

Unckell et al. generated HPV33 pseudovirions by the assembly of VLPs in COS-7 cells containing multiple copies of a marker plasmid [34]. In addition, HPV18 pseudovirions were prepared using recombinant vaccinia viral expression vectors that were transfected into mammalian 293T cells [35]. This type of pseudovirion was shown to be infectious, as it could transfer $\beta$-galactosidase activity or confer resistance to puromycin to a number of cell types when the pseudovirions encapsidated plasmids containing either the $\beta$-galactosidase gene or the puromycin-resistance gene, respectively. This finding indicated that intracellular episomal DNAs of suitable sizes can be encapsidated by the HPV18 L1 and L2 proteins without the need for any HPV packaging signal, facilitating the infection of other cells. This finding facilitated the development and refinement of subsequent 
pseudovirion neutralization systems. Kawana et al. developed a cell-free system for generating infectious HPV16 pseudovirions [36]. The HPV16 capsid proteins L1 and L2 were expressed and co-assembled into VLPs in insect cells (Sf9). The HPV16 VLPs were then disassembled in the presence of the reducing agent, 2-mercaptoethanol and reassembled by removal of the reducing agent in the presence of a $\beta$-galactosidase expression plasmid. The in vitro construction of this pseudovirion system with marker plasmids would be potentially useful in developing an assay for evaluating virus-neutralizing antibodies from animal or human sera. Coursaget and colleagues also used the similar disassembly/assembly method on VLPs isolated from insect cells $(\mathrm{Sf} 21)[37,38]$. The marker genes were then encapsidated in the process of disassembly/assembly. Another study by Zhao et al. suggested that the packaging of the genome within papillomavirus pseudovirions also involves the interaction of the L2 protein with specific DNA sequences and these authors also demonstrated that the PsV capsid has the potential to encapsidate plasmids up to $10.2 \mathrm{~kb}$ in size [39].

Rossi et al. utilized Saccharomyces cerevisiae to generate HPV16 pseudovirions [40]. In addition to the plasmid encoding the HPV16 capsid proteins, another target plasmid containing the green fluorescent protein gene was used to monitor the delivery of the plasmid into mammalian cells upon infection. The reporter gene expression could be analyzed by fluorescence activated cell sorting in vitro and detected by confocal microscopy in vivo. Thus HPV16 pseudovirions produced in yeast may be useful for both in vitro transduction and in vivo gene delivery. Buck et al. chose secreted alkaline phosphatase (SEAP) as the reporter molecule with signal 
amplification in the presence of an appropriate enzymatic substrate [41]. The SEAP reporter plasmid which contains the SV40 origin of replication and codon-modified papillomavirus capsid genes, L1 and L2, were co-transfected into a 293 cell line, 293TT, that was engineered to express high levels of SV40 large tumor antigen (LT) to produce high-titer pseudovirions. The LT antigen unwinds DNA containing the SV40 origin of replication and initiates reporter plasmid replication. High titers (at least $10^{6}$ pseudovirions from a $75 \mathrm{~cm}^{2}$ flask of cells) can be routinely achieved due to the presence of SV40 origin of replication. The PsV encapsidating the SEAP reporter plasmid was used to develop a high-throughput in vitro neutralization assay with a 96 well plate format. Through this method, antibody-mediated PsV neutralization is detected by a reduction in SEAP activity. The SEAP activity was monitored using a highly sensitive chemiluminescent reporter system. This PsV-based neutralization assay was amenable for routine assays of large numbers of clinical samples with desired high throughput and high sensitivity due to enzyme turnover and chemiluninescence detection [26]

To further improve sensitivity, Bousarghin et al. developed a new method of HPV VLP-DNA complex formation to generate HPV16 and HPV31 pseudovirions [42]. This method was also sensitive for detecting the very low amount of neutralization antibodies after natural infection. It was also suitable for testing neutralization antibodies from human sera after vaccination.

Because the mechanism of a successful L1-based vaccine presumably relies on the in vitro generation of functional antibodies, the development and improvement of

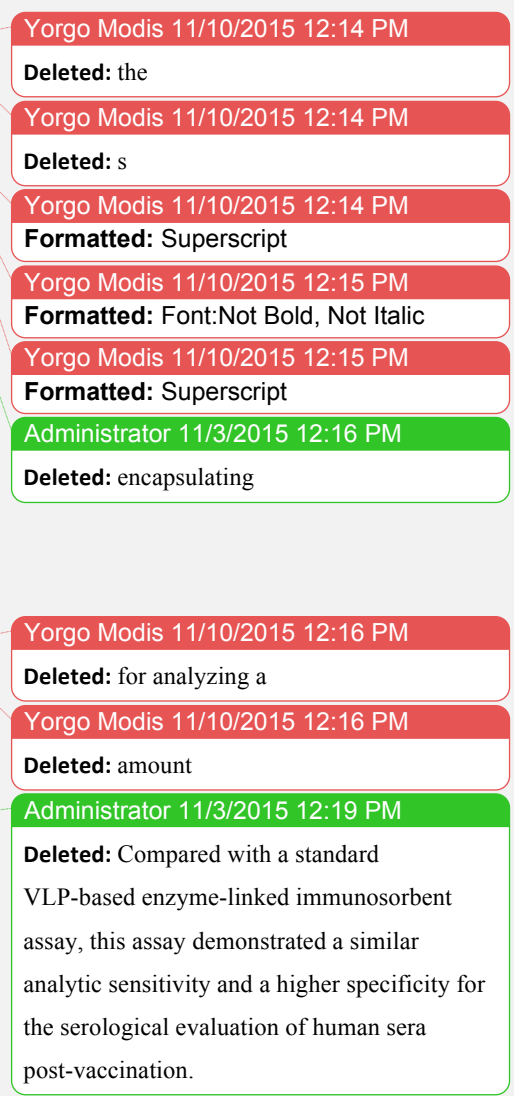

Administrator 10/22/2015 9:37 PM Deleted: vivo 
PsV-based neutralization assays has enabled the implementation of this assay in evaluation of neutralization antibodies after both immunization and natural infection.

\section{Assessment of the neutralization activity of HPV antibodies}

Prophylactic vaccines confer protection mainly through the elicitation of neutralizing antibodies. Therefore, the evaluation of neutralizing antibody levels is highly desirable for predicting vaccine efficacy. The development of in vitro HPV PsV-based neutralization assays has gradually met the need for the functional evaluation of human or animal serum samples post-vaccination. The PsV-based neutralization assay has been highly effective for quantifying potentially protective antibody responses against HPV acquired through natural infection and in prophylactic vaccine studies.

Yeager et al. demonstrated that HPV pseudovirions with $\beta$-lactamase as a reporter constitute a novel and efficient approach to detect and characterize HPV neutralizing antibodies. The results showed that no significant differences were observed between the HPV11 athymic mouse neutralization assay and the HPV11 PsV-based neutralization assay using a panel of sera samples with a range of titers differing by more than 300-fold. This also indicated that the PsV-based assay could detect the majority of functional antibodies elicited with the VLP, immunization. The PsV-based neutralization assays were predominantly type specific for the tested serum.

Significant cross-reactivity could only be observed between the closely types at the lowest serum dilution (1:100) such as HPV6 and HPV11 [27]. Pastrana et al. 
developed a sensitive high-throughput neutralization assay that was based on pseudovirions encapsidating a SEAP reporter gene [26]. This neutralization assay has been validated for HPV16 and HPV18 by analyzing the neutralization titers in serum samples of VLP-vaccinated women and women naturally infected with HPV. The results demonstrated that SEAP-based HPV neutralization assays directly measured the activity of antibodies relevant to vaccine efficacy and natural infection studies. Additionally, the HPV16 PsV-based neutralization assay is more sensitive than ELISA method when assessing antibodies in sera from unvaccinated women. The greater sensitivity was demonstrated by testing the neutralization activity of the sera from the individuals who were HPV16 DNA positive but HPV16 ELISA negative. Krajden et al. prepared the HPV16 and HPV18 PsVs using red fluorescent protein as a reporter [25]. They utilized this system to analyze the prevalence of HPV16 and HPV18 neutralization antibody levels in sera from prenatal pregnant women. Furthermore, HPV16 and HPV18 type-specific neutralizing antibodies from natural infection can be reliably measured and quantitated by the PsV-based neutralization assay. In addition, with the specificity of neutralizing antibody detection, the assay could be used in epidemiological investigation for the prior exposure population to vaccine types in order to optimize the use of the vaccine and monitor the antibody response induced by the vaccine.

The PsV-based neutralization assay is more reliable than the VLP-based ELISA as a measure of neutralizing antibodies. The PsV-based assay usually measures the

\section{User 11/9/2015 12:53 PM}

Deleted: With detection of neutralizing, compared to HPV16 ELISA, the results suggested that the

\begin{tabular}{l} 
Yorgo Modis 11/10/2015 12:41 PM \\
Deleted: ies \\
User 11/9/2015 12:54 PM \\
Deleted: population \\
Yorgo Modis 11/10/2015 12:42 PM \\
Deleted: $\mathrm{n}$ \\
\hline User 11/9/2015 12:55 PM \\
\hline Deleted: in \\
Yorgo Modis 11/10/2015 12:42 PM \\
Deleted: indication \\
Yorgo Modis 11/10/2015 12:43 PM \\
Deleted: is \\
Yorgo Modis 11/10/2015 12:43 PM \\
Deleted: was \\
\hline
\end{tabular}


To improve the type coverage and amenability for automation, Sehr et al.

developed a high-throughput and automated neutralization assay with improved sensitivity for HPV16, 18, 31, 45, 52, 58 and BPV1 [43]. To fulfill a high throughput of the assay, the assay plate, preparation was separated from the readout of the neutralization assay. Large batches of 384-well assay plates with serially diluted serum samples were prepared by an automated liquid handling system. This jmproved the throughput of the assay, making it more amenable for further automation. In addition, authors utilized the luciferase as the reporter. Compared to the SEAP, the use of the luciferase increased the sensitivity of the assay with higher signal to background ratios. The analytic sensitivity has been demonstrated for analyzing the World Health Organization international antibody standards in sera for HPV16 and HPV18. Accordingly, increased robustness and decreased assay variation was demonstrated, in addition to its improved sensitivity.

The correlation between the PsV-based neutralization assay and other serological assays used to evaluate the neutralizing antibodies was also investigated. Brown et al. assessed the correlations between the PsV-based neutralization assay and the competitive Luminex immunoassay and immunoglobulin G Luminex immunoassay [44]. In this assessment, subsets of serum samples were selected from three prior clinical trials of the quadrivalent HPV vaccine. The results showed that the three assays gave results that were highly concordant and reflected measurement of neutralization antibodies (or functional titer). Robbins et al. also compared the SEAP

\section{User 11/9/2015 12:54 PM}

Deleted: or natural infection

\begin{tabular}{l} 
Yorgo Modis 11/10/2015 12:25 PM \\
Deleted: authors separated \\
\hline Yorgo Modis 11/10/2015 12:25 PM \\
Deleted: $\mathrm{s}$ \\
\hline Yorgo Modis 11/10/2015 12:26 PM \\
\hline Deleted: and \\
\hline Yorgo Modis 11/10/2015 12:26 PM \\
\hline Deleted: performance \\
\hline Yorgo Modis 11/10/2015 12:27 PM \\
\hline Deleted: Preparation of 1 \\
\hline Yorgo Modis 11/10/2015 12:27 PM \\
\hline Deleted: was \\
\hline Yorgo Modis 11/10/2015 12:26 PM \\
\hline Deleted: accomplished \\
\hline Yorgo Modis 11/10/2015 12:27 PM \\
\hline Deleted: Therefore, t \\
\hline Yorgo Modis 11/10/2015 12:27 PM \\
\hline Deleted: also \\
\hline
\end{tabular}


neutralization assay with both the competitive Luminex immunoassay (cLIA) and the VLP-coating based ELISA [45]. The data demonstrated a good correlation among these assays, Krajden et al. made another assessment of the HPV16 and HPV18 antibody response by the pseudovirion neutralization assay, Merck competitive Luminex (Merck cLIA) and Merck total IgG Luminex (Merck TIgG) immunoassays in a reduced dosage quadrivalent HPV vaccine trial [46]. The correlation between the PsV-based neutralization assay and cLIA was better for HPV18 than for HPV16, whereas the correlation between the PsV-based neutralization assay and total IgG was similar for both of the viral types. This assessment indicated that the PsV-based neutralization assay was more sensitive than cLIA and likely more specific than the total IgG immunoassay.

\section{Although good correlation among different assays has been demonstrated, the cLIA,} SEAP-NA, ELISA are biologically and technically different assays [45, 47]. They measure the antibody response of serum in different aspects. The cLIA assay developed by Merck was mainly used in the clinical trials to measure all the neutralizing antibodies of all $\operatorname{IgG}$ classes in serum samples that compete for binding to a specific epitope on VLP surface, e.g. V5 for HPV16, J4 for HPV18 with an indication of a binding fluorescent signal strength. This assay is highly type specific. Therefore, in a single assay the individual reactivity to multiple VLPs can be simultaneously detected owing to the multiplexing capacity of the Luminex platform. However, the major disadvantage is that it measures only the neutralizing antibodies that bind the specific epitope, which make up a subset of the total antibodies elicited. 
Thus, this assay may underevaluate the potentially protective antibody response induced by the vaccine, which are non-overlapping with the tracing antibody.

SEAP-NA reflects the overall serum neutralizing antibodies by measuring a reporter gene product. This assay is considerably more laborious but recently it has been developed to be highly throughput and highly sensitive. Therefore, this assay is used primarily to correlate with the clinical protection potential of the serum samples.

The ELISA developed by GSK measures the antibodies that bind to a VLP antigen fixed on a solid face. Conformational heterogeneity of the surface immobilized antigen is an inevitable issue for plate based ELISAs. The signal was detected by adding a secondary antibody conjugated with an enzyme e.g. alkaline phosphatase. It has the disadvantage of potentially detecting the nonneutralizing antibodies elicited by the VLPs,

The PsV-based neutralization assay is still the gold standard among various assays for evaluating the potentially protective antibodies, The implementation of the PsV-based neutralization assay is of the utmost importance in the evaluation of clinical serum samples to demonstrate the efficacy of prophylactic HPV vaccines.

Comparison and correlation could be better determined by the first antibody standard established by WHO Expert Committee on Biological Standardization [48]

\section{Different binding sites for neutralizing antibodies to the HPV capsid}

Natural infection with HPV or vaccination with HPV L1 VLP-based vaccines can induce a complex antibody response. A wide array of different antibodies directed to

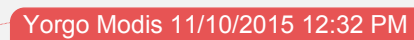

Deleted: the results obtained from Yorgo Modis 11/10/2015 12:32 PM

Deleted: are

Yorgo Modis 11/10/2015 12:32 PM

Deleted: most truly

Deleted: efficacy of neutralizing antibodies 
different epitopes presented on the VLP surface can be elicited by the virions or

VLP-based immunogens. The neutralizing antibodies usually exert their function by

blocking the binding and entry of HPVs into host cells. HPV16 binding to heparin

sulfate proteoglycans (HSPGs) on the basement membrane was firstly reported by

Johnson et al. using a murine cervicovaginal challenge model in vivo, Additionally,

initial HPV16 binding occurs via heparin sulfate proteoglycans (HSPGs) located

either on the epithelial cell surface or on the basement membrane was also verified in

vitro by Knappe et al. and Richards et al. [49-51]. HSPGs were first described as HPV

binding receptors using HPV11 L1 VLP on HaCaT cells $[52,53]$. The other studies

showed that HSPGs were essential for infection using HPV16 PsV and HPV33 PsV

on COS-7 cells $[49,54]$. The exterior surface of HPVs is mainly composed of the L1

capsid protein with 5 surface loops. HPV16 L1 residues Lys278 and Lys361 on the

top of the pentamer were identified as the primary attachment sites to HSPGs, which

demonstrated a direct interaction between HPV16 L1 and HSPGs [50]. Thus, the

interaction between neutralizing antibodies and the major capsid protein can block the

virions from binding to the target host cells, thus inhibiting the subsequent cell entry

process.

Various neutralizing mAbs have been isolated by different labs to further elucidate

the neutralization mechanism and the specific binding sites of neutralizing mAbs.

Three different binding modes for neutralization antibodies on the viral capsid have

been determined to date (Figure 2). Bovine papillomavirus and human papillomavirus are in the same genus and are highly similar to each other, particularly in regards to

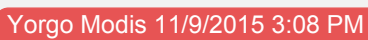

Deleted:

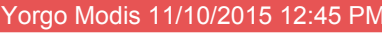

Deleted: .

Administrator 10/23/2015 11:59 AM

Deleted: Initial HPV16 binding occurs via heparin sulfate proteoglycans (HSPGs) located either on the epithelial cell surface or on the basement membrane 
their capsid surface structure [55]. Therefore, BPV has commonly been used as a surrogate to study the biological profile of HPV. Two immunocomplexes of the BPV1 major capsid protein L1 and two neutralizing mAbs were analyzed by electron cryomicroscopy (cryoEM) and three-dimensional image reconstruction to $13 \AA$ resolution [56]. $\mathrm{mAb} \# 9$ is representative of a set of neutralization antibodies that can inhibit viral binding to the host cell surface. In the cryoEM structure, mAb \#9 bound to the major L1 molecules of both pentavalent and hexavalent capsomeres. The epitope of $\mathrm{mAb} \# 9$ was located in the outer tips of capsomeres (Figure 2). The other neutralizing antibody, $\mathrm{mAb} 5 \mathrm{~B} 6$, can efficiently neutralize the papillomaviruses without significantly inhibiting viral binding to the host cell surface. Thus, this type of neutralizing antibody may neutralize the virus by preventing capsid uncoating and thus blocking the release of viral DNA into the nucleus. Structural analysis showed that mAb 5B6 did not bind to pentavalent capsomeres, which reflected the significant structural and environmental difference of its epitope between the pentavalent and hexavalent capsomeres_[56]. A similar observation was made for the different conformations of pentavalent versus hexavalent capsomeres. The epitope localization showed that mAb 5B6 bound both monovalently and bivalently to the sides of hexavalent capsomeres approximately two-thirds of the way down from the outer tips (Figure 2). Other work on recombinant vaccine VLPs by Zhao et al. demonstrated two different binding models for two different neutralizing antibodies (H11.B2 for HPV11 and H16.V5 for HPV16) using cryoEM image reconstruction [57, 58]. Both H16.V5 and H11.B2 showed highly efficient neutralization activity in the 
pseudovirion neutralization assay. These antibodies also recognized immuno dominant epitopes when analyzed by competition assay using human sera from naturally infected individuals. The cryoEM structure of the VLP-Fab complex in comparison to VLP alone revealed that the binding site of mAb H11.B2 was located at the center of the capsomere, coincident with the L2 binding sites in the capsid, indicating 72 potential binding sites per capsid [57]. However, the other well-studied mAb H16.V5 with an extremely high neutralization efficiency showed a different binding mode on the apex of the capsomere $[57,59]$. H16.V5 bound only to capsomeres with six neighboring capsomeres (at the 3-fold axes of symmetry), but there was no evidence from the density map for its binding to the pentavalent capsomeres (at 5-fold axes of the symmetry) (Figure 3). Based on the cryoEM structures, there are 300 potential binding sites for mAb H16.V5 due to steric hindrance or different structures between hexavalent versus pentavalent capsomeres in the recombinant icosahedral VLPs.

A recent study by Lee et al. also determined the spatial structure of the immunocomplex of H16.V5 and HPV16 pseudovirions [59]. The complete epitope of H16.V5 was identified using cryoEM and 3D image reconstruction. Based on the experimental methods using the model of VLP-Fab complexes, the H16.V5 epitope was found to be composed of the antigenic loops $\mathrm{BC}, \mathrm{DE}$ and $\mathrm{EF}$, in addition to the previously identified epitopes located in the FG loop and HI loop. The study indicated that the binding of H16.V5 Fab induced a global conformational change. The study showed that the H16.V5 Fab bound to the hexavalent capsomeres as well as to the 
pentavalent capsomeres. H16.V5 Fab also bound preferentially to the hexavalent capsomeres over the pentavalent capsomeres (Figure 3). This preferred binding suggested that the affinity for the two binding sites was different and that this phenomenon might exist intrinsically or be induced by H16.V5 Fab binding. In addition, structural consolidation in the base of the capsomere as well as on the surface flexible loop was observed by the binding of H16.V5 to the capsid (Figure 3).

The other recent study by Guan et al. showed that Fab fragment from three additional HPV16-specific antibodies, mAbs H16.1A H16.14J and H263.A2, had complex and overlapping epitopes similar to the H16.V5 epitope [61]. CryoEM image reconstructions showed that the $\mathrm{DE}$ loop formed the core of each $\mathrm{mAb}$ footprint, and that the mAbs also bound to the FG, HI, DE and EF loops, although the participation of the latter loops varied between the antibodies (Figure 3).

In addition to the roles played by major capsid protein L1, the minor capsid protein L2 was also determined to be involved in the viral cell entry process, a critical step for viral infection. A subtle conformational change is triggered by the interaction between HSPGs and the HPV capsid that further leads to the exposure of an amino-terminal portion of L2. Subsequently, the cleavage of L2 by furin is required for further infection, which results in additional changes to the capsid conformation. More importantly, during this furin cleavage process, a broadly neutralizing epitope of the L2 protein is exposed. The exposure of this epitope was first characterized with the neutralizing mAb RG-1 [22, 62].

The observation of different binding modes for various neutralization antibodies 
against the major capsid protein L1 or the minor capsid protein L2 at a higher resolution would shed light on the mechanisms of how HPV virions could be neutralized during cell attachment or entry. This will likely soon be achievable with the improvement of direct electron detectors and image reconstruction software in cryoEM.

The encouraging clinical trial results and long-term follow-up results have validated the efficacy of the launched vaccines (Cervarix ${ }^{\circledR}, \operatorname{Gardasil}^{\circledR}$ and Gardasil ${ }^{\circledR} 9$ ). The protective immunity conferred by vaccination is due to the presence of functional epitopes in the vaccine antigens. Neutralizing antibody responses are believed to be the most direct evidence of vaccine efficacy and the primary mechanism of vaccine-induced protective immunity [63]. Therefore, integrity of the correct spatial conformation and sufficient exposure of the functional epitopes are essential during the bioprocessing of vaccine antigens. Some epitope-based assays were designed and developed to evaluate antibody response in clinical sera to verify vaccine immunogenicity. Secreted alkaline phosphatase neutralization assay has been used to quantify the neutralizing antibody response elicited by Cervarix ${ }^{\circledR}$ and $\operatorname{Gardasil}_{A}^{\circledR}[12$, 26, 64-67].More recently, serological assays for an E.coli-derived VLP-based vaccine were performed with a slightly different format of PsV-based assay [68-70]. For Gardasil $^{\circledR}$, the competitive Luminex immunoassay was developed to measure neutralizing antibodies with high-sensitivity by competing for binding to one epitope, e.g., H16.V5 for HPV16 and H18.R5 for HPV18. [71-74]. With a better understanding of the functional epitopes, more epitope-based assays will be 
reasonably designed [75]. Therefore, the accurate epitope determination at molecular level will shed light on assay developments to monitor the manufacturing process of vaccines and to evaluate the effective antibody response in clinical sera.

\section{Discussion}

Currently, there are two prophylactic HPV vaccines on the market, the bivalent vaccine containing the VLP antigen for HPV16/18 (Cervarix ${ }^{\circledR}$ from GSK) and the quadrivalent (or recently-licensed nonavalent) vaccine against HPV6, 11, 16, and 18 (with $31,33,45,52,58$ ) from Merck. Both vaccines are based on VLPs as the active component, produced by expressing the L1 protein using recombinant technology. The expressed L1 proteins self-assemble into VLPs that resemble the native virions with specific spatial structural features. This high degree of resemblance of the VLPs to viral capsids is the structural basis for their ability to elicit neutralization and functional antibodies for prevention of viral infection. Two issues are the questions being discussed when licensing a new biosimilar HPV vaccine: a) what is the minimal level of neutralizing antibody required for protection? b) what endpoint(s) should be used for evaluation of a new HPV vaccine-clinical (i.e., CIN2+), persistent infection or virological endpoint? The former question is difficult to address due to the high efficacy of the vaccines. For the latter questions, consensus has been reached gradually among scientists that persistent infection is the pre-requisite for CIN1, CIN2 and higher precancerous lesions, thus, using endpoints other than the initially used "CNI2+" for Gardasil and Cervarix is feasible. Based on what has been learned since 
understanding of the vaccine efficacy and endpoints that can be used for the evaluation of HPV vaccines; persistent infection ( $>6 \mathrm{Mo}$ ) or virological endpoints

(such as virus neutralization activity of serum samples using the PsV-based neutralization assay). This has been reflected in the WHO guidelines for licensing existing or new HPV vaccines in their countries/regions by the respective national regulatory agencies [Recommendations to assure the quality, safety and efficacy of recombinant human papillomavirus virus-like particle vaccines. WHO Expert Committee On Biological

Standardization 2015:

http://www.who.int/biologicals/BS2252 HPV Recommendations 30062015 tz.pdf? ua $=30062011$.

The presence of functional neutralizing antibodies is the basis for conferring protection against HPV infection, Neutralizing antibodies played a key role in preventing HPV infection by blocking attachment to cells or inhibiting the cell entry process depending on the specific epitopes on the viral capsid they recognize. The major capsid protein, L1, harbors most of the neutralizing epitopes. This is also the basis for the function of the currently licensed L1-based vaccines. However, some reports showed some neutralizing Abs could be elicited by recombinant L2 protein. Since L2 has higher degree of amino acid sequence homology as compared to L1, it is also a target for broad spectrum vaccine development. Since most PsV systems contain both L1 and L2, therefore the same PsV-based neutralization assay can be used to assess the function of the Abs elicited by either L1- or L2-based vaccines. In addition, the PsV neutralization assay could provide an alternative method in
Yorgo Modis 11/9/2015 2:59 PM

Deleted: which

Yorgo Modis 11/9/2015 2:59 PM

Deleted: .

Yorgo Modis 11/9/2015 2:59 PM

Deleted: The

Yorgo Modis 11/9/2015 2:59 PM

Deleted: the

Yorgo Modis 11/9/2015 2:59 PM

Deleted: i.e., the

Yorgo Modis 11/9/2015 3:00 PM

Deleted: the

Yorgo Modis 11/10/2015 1:30 PM

Formatted: Highlight

Yorgo Modis 11/10/2015 1:30 PM

Formatted: Highlight

Yorgo Modis 11/10/2015 1:30 PM

Formatted: Highlight

Yorgo Modis 11/9/2015 3:01 PM

Deleted: - cite the WHO guidelines Oct2015 User 11/9/2015 12:18 PM

Deleted: In HPV infection, the type-specific immune response is mainly directed to the $\mathrm{L} 1$ protein, the major capsid protein. The VLP-based prophylactic HPV vaccine mainly elicited effective humoral responses for preventing HPV infection. The efficacy of the vaccines is dependent upon the integrity of type-specific conformational epitopes on the antigen surface. Numerous preclinical trials demonstrated that the vaccines were highly immunogenic and efficacious against viral infection, with high levels of functional antibodies induced. For instance, the quadrivalent vaccine has been evaluated in two Phase III studies, FUTURE I and FUTURE II,

and the bivalent vaccine has also been evaluated in two Phase III trials, PATRIF User 11/9/2015 12:28 PM

Deleted: with different neutralization mechanisms User 11/9/2015 12:31 PM

Deleted: User 11/9/2015 12:30 PM

Deleted: It is generally accepted that HPV first binds to the heparin sulfate proteoglycan ...[3] 
bioprocessing for VLP quality analysis for assuring the presence of neutralization epitopes on antigens. This analysis can be carried out with in vivo mouse potency assays. Mouse potency was used to evaluate the immunogenicity of vaccines with the indication of the $\mathrm{ED}_{50}$ value. Specifically, mice were immunized with serially diluted vaccine and the serum samples were collected four weeks after immunization.

Subsequently, the anti-vaccine antigen antibodies were detected in an antigen-coating

ELISA assay. The $\mathrm{ED}_{\mathbb{R}_{0}}$ value was calculated with indication of the \{something missing here 3 that would result in $50 \%$ seroconversion for the tested animals [82] The titer of neutralization antibodies reflected of the level of functional Abs elicited by immunization with immunogens harboring, conformational and functional epitopes. For many infectious diseases, assays to detect neutralizing antibodies are used to assess the immunogenicity of prophylactic vaccines. In vitro PsV-based neutralization assays involve measuring the inhibition of HPV pseudovirion binding and infection of cultured cells by employing a reporter gene to score the level of infection.

Due to the type-specificity of the current L1 VLP-based vaccines, a broad-spectrum next generation HPV vaccine needs to be developed that would prevent the infection of more additional HPV types for broader type coverage. A nonavalent vaccine, with L1 antigens of HPV6, 11, 16, 18, 31, 33, 45, 52, and 58, was recently licensed in the USA (Gardasil ${ }^{\circledR} 9$ developed by Merck) [83-85]. The HPV capsid contains the major $\mathrm{L} 1$ protein and the minor L2 protein. The L1 protein is abundantly present on the viral surface, which harbors the immunodominant epitopes inducing strong B cell

\begin{tabular}{|l|}
\hline Yorgo Modis 11/10/2015 12:19 PM \\
Deleted: In detail \\
Yorgo Modis 11/10/2015 12:19 PM \\
Formatted: Subscript \\
Yorgo Modis 11/10/2015 12:19 PM \\
\hline Deleted: the \\
\hline Yorgo Modis 11/10/2015 12:19 PM \\
\hline Deleted: which had been serially diluted \\
\hline Yorgo Modis 11/10/2015 12:20 PM \\
\hline Deleted: 4 \\
\hline Yorgo Modis 11/10/2015 12:20 PM \\
\hline Formatted: Subscript \\
\hline Yorgo Modis 11/10/2015 1:30 PM \\
\hline Formatted: Highlight \\
\hline Yorgo Modis 11/10/2015 12:22 PM \\
\hline Comment [1]: Qinjian, there is at least one \\
work missing here... \\
\hline Yorgo Modis 11/10/2015 1:30 PM \\
\hline Formatted: Highlight \\
\hline User 11/9/2015 12:37 PM \\
\hline Deleted: With this assay, the results reflect t \\
\hline User 11/9/2015 12:38 PM \\
\hline Deleted: s \\
\hline User 11/9/2015 12:39 PM \\
\hline Deleted: capable of binding to \\
\hline
\end{tabular}


responses. However, the L2 protein is barely visible to the immune system. Only when the virus binds to the basement membrane of host cell does L2 become exposed due to conformational changes in the capsid[50,86]. Its neutralization epitopes are deemed to be transiently exposed after furin cleavage in the viral infection process. The process was induced by the interaction between L1 and HSPGs that triggers a conformational change of the capsid. It is the transient exposure that induces the immune response to generate the specific antibodies. Several cross-neutralizing antibodies have been identified and studied extensively, such as RG1 recognizing residues 17-36 of L2 and the other two antibodies K4L2 and K18L2 targeting the sites located at residues 20-38 of L2, which are exposed after furin cleavage [22-24].Therefore, the L2 protein is also a candidate for a broad-spectrum HPV prophylactic vaccine with the conserved epitopes located at the N-terminus of the L2 protein. Recently, efforts have been made to develop an L2-based prophylactic vaccine for the development of the next generation of HPV vaccine, which has had some success [87-91].

Understanding of the clinically relevant epitopes is critical for vaccine design and quality control in the manufacturing process of vaccines. The mAbs recognizing immuno dominant epitopes are useful molecular tools. The binding activity to the neutralizing mAbs can serve as a surrogate marker for VLP antigens. Therefore, mAbs can be utilized for process monitoring and product assessment to ensure the product consistency for VLP-based vaccines. More specifically, the entire conformational epitope recognized by the mAb HPV16.V5 was recently identified by

\begin{tabular}{l} 
Yorgo Modis 11/9/2015 3:09 PM \\
Deleted: just \\
\hline Yorgo Modis 11/9/2015 3:10 PM \\
\hline Deleted: well-characterized and \\
\hline Yorgo Modis 11/10/2015 12:46 PM \\
\hline Formatted: Font:Not Bold, Not Italic \\
\hline Yorgo Modis 11/9/2015 3:10 PM \\
\hline Deleted: the aa \\
\hline Yorgo Modis 11/9/2015 3:11 PM \\
\hline Deleted: aa \\
\hline Yorgo Modis 11/9/2015 3:11 PM \\
\hline Deleted: N-terminus \\
\hline Administrator 11/2/2015 10:45 PM \\
\hline Deleted: This transient exposure could enable \\
the elicitation of an anti-L2 neutralizing \\
antibody response \\
\hline Administrator 11/2/2015 10:26 PM \\
\hline Deleted: \\
\hline User 11/9/2015 12:43 PM \\
\hline Deleted: of vaccine production \\
\hline \\
\hline
\end{tabular}


Lee et al., and this work also advanced the understanding of the neutralization mechanism of the well-studied HPV16.V5 [59]. This mAb was also used in the clinical serology assay for Gardasil ${ }^{\circledR}$ in the competitive Luminex-based immunoassay, where the effectiveness of competition of the serum samples with H16.V5 was used as a surrogate marker for vaccine efficacy against HPV16 [45, 60, 71-73]. The binding of HPV16.V5 to the capsid resulted in a significant conformational change and structural consolidation that altered the capsid-Ab complex. This binding ordered the apical loops of HPV16 and consolidated the "invading-arm" structure. In addition, this conformational change was transmitted to the lower region of the capsomere and tightened the intercapsomeric connection at the capsid floor.

A similar phenomenon of conformational changes induced by mAb binding was also observed in other viral capsid-Ab complexes. The neutralizing mAb 1A1D-2 strongly neutralized dengue virus types 1,2 , and 3 . The binding of this $\mathrm{mAb}$ to the envelope glycoprotein $\mathrm{E}$ of dengue virus altered the arrangement of the surface glycoproteins. The Fab molecules might capture the transient viral intermediate conformation, further hiding the 1A1D-2 epitopes exposed for Fab binding. This change is likely the potential mechanism by which this neutralization antibody could inhibit viral attachment to host cells [1]. Another example is the binding of neutralization mAb E18 to the mature human enterovirus 71 (EV71), which induces the conformational change in the capsid. mAb E18 recognized the conformation of empty and immature particles of EV71, which are similar to the "A" particles when the EV71 recognizes a potential host cell before genome release. The binding of E18 
to mature virions induced a conformational change that transformed the infectious virions into "A" particles. This mechanism demonstrated that mAb E18 could neutralize the virus by inducing genome release. Therefore, such a characteristic made the E18 a potential therapeutic Ab candidate [2].

In summary, the PsV-based neutralization system has been broadly used in the efficacy evaluation of neutralization antibodies generated by vaccination or natural infection. Antibodies could exert their viral neutralizing functions via different mechanisms. These include blocking the binding between viruses and cell receptors and altering the epitopes recognized by the cell receptors by inducing conformational changes in the viral capsid. Three binding modes for HPV capsid and mAb were determined using cryoEM image reconstruction techniques at intermediate resolution. Higher resolution structural determination will aid in the understanding of the epitope details and the functional mechanism of neutralization mAbs. In addition, better knowledge regarding the mAb neutralization mechanism will advance process understanding and process control during bioprocessing in vaccine production and in the design and development of the next generation vaccines [30]. 


\section{Acknowledgements}

The authors acknowledge the funding support from the Chinese government: National 863 Program of China (2014AA021302), National Natural Science Fund (81373061 and 81471934), Fujian Provincial Program for Construction Plan of Science and Technology Innovation Platform (2014Y2101). This work was also supported by a Senior Research Fellowship from the Wellcome Trust, grant number 101908/Z/13/Z, to Y.M.

Author Contributions: X.Z., S.L., Y.M., Z.L., J.Z., N.X. and Q.Z. wrote the paper.

Conflicts of Interest: The authors declare no conflict of interest. 


\section{References}

1. Lok SM, Kostyuchenko V, Nybakken GE, et al. Binding of a neutralizing antibody to dengue virus alters the arrangement of surface glycoproteins. Nat Struct Mol Biol 2008; 15: 312-317. DOI: $10.1038 /$ nsmb.1382

2. Plevka P, Lim PY, Perera R, et al. Neutralizing antibodies can initiate genome release from human enterovirus 71. Proc Natl Acad Sci U S A 2014; 111: 2134-2139. DOI: 10.1073/pnas.1320624111

3. Christensen ND, Cladel NM, Reed CA, et al. Hybrid papillomavirus L1 molecules assemble into virus-like particles that reconstitute conformational epitopes and induce neutralizing antibodies to distinct HPV types. Virology 2001; 291: 324-334. DOI: 10.1006/viro.2001.1220

4. Dale CJ, Liu XS, De Rose R, et al. Chimeric human papilloma virus-simian/human immunodeficiency virus virus-like-particle vaccines: immunogenicity and protective efficacy in macaques. Virology 2002; 301: 176-187.

5. Tyler M, Tumban E, Peabody DS, Chackerian B. The use of hybrid virus-like particles to enhance the immunogenicity of a broadly protective HPV vaccine. Biotechnol Bioeng 2014; 111: 2398-2406. DOI: 10.1002/bit.25311

6. zur Hausen H. Papillomaviruses and cancer: from basic studies to clinical application. Nat Rev Cancer 2002; 2: 342-350. DOI: 10.1038/nrc798

7. Arbyn M, Tommasino M, Depuydt C, Dillner J. Are 20 human papillomavirus types causing cervical cancer? J Pathol 2014; 234: 431-435. DOI: 10.1002/path.4424

8. Jin L, Xu ZX. Recent advances in the study of HPV-associated carcinogenesis. Virol Sin 2015; 30: 101-106. DOI: 10.1007/s12250-015-3586-3

9. Buck CB, Day PM, Trus BL. The papillomavirus major capsid protein L1. Virology 2013; 445: 169-174. DOI: 10.1016/j.virol.2013.05.038

10. Wang JW, Roden RB. L2, the minor capsid protein of papillomavirus. Virology 2013; 445: 175-186. DOI: 10.1016/j.virol.2013.04.017

11. Kirnbauer R, Booy F, Cheng N, Lowy DR, Schiller JT. Papillomavirus L1 major capsid protein self-assembles into virus-like particles that are highly immunogenic. Proc Natl Acad Sci U S A 1992; 89: 12180-12184.

12. Einstein $\mathrm{MH}$, Baron $\mathrm{M}$, Levin $\mathrm{MJ}$, et al. Comparison of the immunogenicity and safety of Cervarix and Gardasil human papillomavirus (HPV) cervical cancer vaccines in healthy women aged 18-45 years. Hum Vaccin 2009; 5: 705-719.

13. Markowitz LE, Hariri S, Lin C, et al. Reduction in human papillomavirus (HPV) prevalence among young women following HPV vaccine introduction in the United States, National Health and Nutrition Examination Surveys, 2003-2010. J Infect Dis 2013; 208: 385-393. DOI: 10.1093/infdis/jit192

14. Deschuyteneer M, Elouahabi A, Plainchamp D, et al. Molecular and structural characterization of the $\mathrm{L} 1$ virus-like particles that are used as vaccine antigens in Cervarix, the ASO4-adjuvanted HPV-16 and -18 cervical cancer vaccine. Hum Vaccin 2010; 6: 407-419.

15. Dunne EF, Naleway A, Smith N, et al. Reduction in Human Papillomavirus Vaccine Type Prevalence Among Young Women Screened for Cervical Cancer in an Integrated US Healthcare Delivery System in 2007 and 2012-2013. J Infect Dis 2015. DOI: 10.1093/infdis/jiv342

16. Dochez C, Bogers JJ, Verhelst R, Rees H. HPV vaccines to prevent cervical cancer and genital warts: an update. Vaccine 2014; 32: 1595-1601. DOI: 10.1016/j.vaccine.2013.10.081

17. Bryan JT. Developing an HPV vaccine to prevent cervical cancer and genital warts. Vaccine 2007; 
25: 3001-3006. DOI: 10.1016/j.vaccine.2007.01.013

18. Villa LL, Costa RL, Petta CA, et al. High sustained efficacy of a prophylactic quadrivalent human papillomavirus types 6/11/16/18 L1 virus-like particle vaccine through 5 years of follow-up. $\mathrm{Br} \mathrm{J}$ Cancer 2006; 95: 1459-1466. DOI: 10.1038/sj.bjc.6603469

19. Kemp TJ, Garcia-Pineres A, Falk RT, et al. Evaluation of systemic and mucosal anti-HPV16 and anti-HPV18 antibody responses from vaccinated women. Vaccine 2008; 26: 3608-3616. DOI: 10.1016/j.vaccine.2008.04.074

20. Day PM, Kines RC, Thompson CD, et al. In vivo mechanisms of vaccine-induced protection against HPV infection. Cell Host Microbe 2010; 8: 260-270. DOI: 10.1016/j.chom.2010.08.003

21. Kemp TJ, Hildesheim A, Safaeian M, et al. HPV16/18 L1 VLP vaccine induces cross-neutralizing antibodies that may mediate cross-protection. Vaccine 2011; 29: 2011-2014. DOI: 10.1016/j.vaccine.2011.01.001

22. Gambhira R, Karanam B, Jagu S, et al. A protective and broadly cross-neutralizing epitope of human papillomavirus L2. J Virol 2007; 81: 13927-13931. DOI: 10.1128/JVI.00936-07

23. Rubio I, Seitz H, Canali E, et al. The N-terminal region of the human papillomavirus L2 protein contains overlapping binding sites for neutralizing, cross-neutralizing and non-neutralizing antibodies. Virology 2011; 409: 348-359. DOI: 10.1016/j.virol.2010.10.017

24. Wang D, Li Z, Xiao J, et al. Identification of Broad-Genotype HPV L2 Neutralization Site for Pan-HPV Vaccine Development by a Cross-Neutralizing Antibody. PLoS One 2015; 10: e0123944. DOI: 10.1371/journal.pone.0123944

25. Krajden M, Karunakaran K, So S, et al. Prevalence of human papillomavirus 16 and 18 neutralizing antibodies in prenatal women in British Columbia. Clin Vaccine Immunol 2009; 16: 1840-1843. DOI: 10.1128/CVI.00238-09

26. Pastrana DV, Buck CB, Pang YY, et al. Reactivity of human sera in a sensitive, high-throughput pseudovirus-based papillomavirus neutralization assay for HPV16 and HPV18. Virology 2004; 321: 205-216. DOI: 10.1016/j.virol.2003.12.027

27. Yeager MD, Aste-Amezaga M, Brown DR, et al. Neutralization of human papillomavirus (HPV) pseudovirions: a novel and efficient approach to detect and characterize HPV neutralizing antibodies. Virology 2000; 278: 570-577. DOI: 10.1006/viro.2000.0674

28. Buck CB, Pastrana DV, Lowy DR, Schiller JT. Efficient intracellular assembly of papillomaviral vectors. J Virol 2004; 78: 751-757.

29. Day PM, Thompson CD, Buck CB, Pang YY, Lowy DR, Schiller JT. Neutralization of human papillomavirus with monoclonal antibodies reveals different mechanisms of inhibition. J Virol 2007; 81: 8784-8792. DOI: 10.1128/JVI.00552-07

30. Zhang $X$, Xin L, Li S, et al. Lessons learned from successful human vaccines: Delineating key epitopes by dissecting the capsid proteins. Hum Vaccin Immunother 2015; 11: 1277-1292. DOI: 10.1080/21645515.2015.1016675

31. Dunne EF, Markowitz LE, Taylor LD, Unger ER, Wheeler CM. Human papilloma virions in the laboratory. J Clin Virol 2014; 61: 196-198. DOI: 10.1016/j.jcv.2014.06.014

32. Zhou J, Sun XY, Stenzel DJ, Frazer IH. Expression of vaccinia recombinant HPV 16 L1 and L2 ORF proteins in epithelial cells is sufficient for assembly of HPV virion-like particles. Virology 1991; 185: 251-257.

33. Roden RBS, Armstrong A, Haderer P, et al. Characterization of a human papillomavirus type 16 variant-dependent neutralizing epitope. J. Virol. 1997; 71: 6247-6252. 
34. Unckell F, Streeck RE, Sapp M. Generation and neutralization of pseudovirions of human papillomavirus type 33. J Virol 1997; 71: 2934-2939.

35. Stauffer Y, Raj K, Masternak K, Beard P. Infectious human papillomavirus type 18 pseudovirions. J Mol Biol 1998; 283: 529-536. DOI: 10.1006/jmbi.1998.2113

36. Kawana K, Yoshikawa H, Taketani Y, Yoshiike K, Kanda T. In vitro construction of pseudovirions of human papillomavirus type 16: incorporation of plasmid DNA into reassembled L1/L2 capsids. J Virol 1998; 72: 10298-10300.

37. Le Cann P, Coursaget $P$, lochmann $S$, Touze A. Self-assembly of human papillomavirus type 16 capsids by expression of the L1 protein in insect cells. FEMS Microbiol Lett 1994; 117: 269-274.

38. Touze A, Dupuy C, Mahe D, Sizaret PY, Coursaget P. Production of recombinant virus-like particles from human papillomavirus types 6 and 11, and study of serological reactivities between HPV $6,11,16$ and 45 by ELISA: implications for papillomavirus prevention and detection. FEMS Microbiol Lett 1998; 160: 111-118.

39. Zhao KN, Sun XY, Frazer IH, Zhou J. DNA packaging by L1 and L2 capsid proteins of bovine papillomavirus type 1. Virology 1998; 243: 482-491. DOI: 10.1006/viro.1998.9091

40. Rossi JL, Gissmann L, Jansen K, Muller M. Assembly of human papillomavirus type 16 pseudovirions in Saccharomyces cerevisiae. Hum Gene Ther 2000; 11: 1165-1176. DOI: 10.1089/10430340050015211

41. Buck CB, Pastrana DV, Lowy DR, Schiller JT. Generation of HPV pseudovirions using transfection and their use in neutralization assays. Methods Mol Med 2005; 119: 445-462. DOI: 10.1385/1-59259-982-6:445

42. Bousarghin L, Combita-Rojas AL, Touze A, et al. Detection of neutralizing antibodies against human papillomaviruses (HPV) by inhibition of gene transfer mediated by HPV pseudovirions. I Clin Microbiol 2002; 40: 926-932.

43. Sehr $\mathrm{P}$, Rubio $\mathrm{I}$, Seitz $\mathrm{H}$, et al. High-throughput pseudovirion-based neutralization assay for analysis of natural and vaccine-induced antibodies against human papillomaviruses. PLoS One 2013; 8: e75677. DOI: 10.1371/journal.pone.0075677

44. Brown $D$, Muller $M$, Sehr $P$, et al. Concordance assessment between a multiplexed competitive Luminex immunoassay, a multiplexed IgG Luminex immunoassay, and a pseudovirion-based neutralization assay for detection of human papillomaviruse types 16 and 18. Vaccine 2014; 32: 5880-5887. DOI: 10.1016/j.vaccine.2014.08.004

45. Robbins HA, Kemp TJ, Porras C, et al. Comparison of antibody responses to human papillomavirus vaccination as measured by three assays. Front Oncol 2014; 3: 328. DOI: 10.3389/fonc.2013.00328

46. Krajden M, Cook D, Yu A, et al. Assessment of HPV 16 and HPV 18 antibody responses by pseudovirus neutralization, Merck cLIA and Merck total IgG LIA immunoassays in a reduced dosage quadrivalent HPV vaccine trial. Vaccine 2014; 32: 624-630. DOI: 10.1016/j.vaccine.2013.09.007

47. Schiller JT, Lowy DR. Immunogenicity testing in human papillomavirus virus-like-particle vaccine trials. J Infect Dis 2009; 200: 166-171. DOI: 10.1086/599988

48. Ferguson $M$, Wilkinson $D E$, Heath $A$, Matejtschuk $P$. The first international standard for antibodies to HPV 16. Vaccine 2011; 29: 6520-6526. DOI: 10.1016/j.vaccine.2011.07.007

49. Knappe M, Bodevin S, Selinka HC, et al. Surface-exposed amino acid residues of HPV16 L1 protein mediating interaction with cell surface heparan sulfate. J Biol Chem 2007; 282: 27913-27922. DOI: 10.1074/jbc.M705127200 
50. Richards KF, Bienkowska-Haba M, Dasgupta J, Chen XS, Sapp M. Multiple heparan sulfate binding site engagements are required for the infectious entry of human papillomavirus type 16. J Virol 2013; 87: 11426-11437. DOI: 10.1128/JVI.01721-13

51. Johnson KM, Kines RC, Roberts JN, Lowy DR, Schiller JT, Day PM. Role of heparan sulfate in attachment to and infection of the murine female genital tract by human papillomavirus. J Virol 2009; 83: 2067-2074. DOI: 10.1128/JVI.02190-08

52. Shafti-Keramat S, Handisurya A, Kriehuber E, Meneguzzi G, Slupetzky K, Kirnbauer R. Different heparan sulfate proteoglycans serve as cellular receptors for human papillomaviruses. J Virol 2003; 77: 13125-13135.

53. Joyce JG, Tung JS, Przysiecki CT, et al. The L1 major capsid protein of human papillomavirus type 11 recombinant virus-like particles interacts with heparin and cell-surface glycosaminoglycans on human keratinocytes. J Biol Chem 1999; 274: 5810-5822.

54. Selinka HC, Giroglou T, Nowak T, Christensen ND, Sapp M. Further evidence that papillomavirus capsids exist in two distinct conformations. J Virol 2003; 77: 12961-12967.

55. Baker TS, Newcomb WW, Olson NH, Cowsert LM, Olson C, Brown JC. Structures of bovine and human papillomaviruses. Analysis by cryoelectron microscopy and three-dimensional image reconstruction. Biophys J 1991; 60: 1445-1456. DOI: 10.1016/S0006-3495(91)82181-6

56. Booy FP, Roden RB, Greenstone HL, Schiller JT, Trus BL. Two antibodies that neutralize papillomavirus by different mechanisms show distinct binding patterns at $13 \mathrm{~A}$ resolution. $\mathrm{J} \mathrm{Mol} \mathrm{Biol}$ 1998; 281: 95-106. DOI: 10.1006/jmbi.1998.1920

57. Zhao Q, Potter CS, Carragher B, et al. Characterization of virus-like particles in GARDASIL(R) by cryo transmission electron microscopy. Hum Vaccin Immunother 2014; 10: 734-739.

58. Zhao Q, Modis $\mathrm{Y}$, High $\mathrm{K}$, et al. Disassembly and reassembly of human papillomavirus virus-like particles produces more virion-like antibody reactivity. Virol J 2012; 9: 52 . DOI: 10.1186/1743-422X-9-52

59. Lee $\mathrm{H}$, Brendle SA, Bywaters SM, et al. A cryo-electron microscopy study identifies the complete H16.V5 epitope and reveals global conformational changes initiated by binding of the neutralizing antibody fragment. J Virol 2015; 89: 1428-1438. DOI: 10.1128/JVI.02898-14

60. Wang Z, Christensen N, Schiller JT, Dillner J. A monoclonal antibody against intact human papillomavirus type 16 capsids blocks the serological reactivity of most human sera. J Gen Virol 1997; 78 ( Pt 9): 2209-2215.

61. Guan J, Bywaters SM, Brendle SA, et al. Structural comparison of four different antibodies interacting with human papillomavirus 16 and mechanisms of neutralization. Virology 2015; 483: 253-263. DOI: 10.1016/j.virol.2015.04.016

62. Gambhira R, Jagu S, Karanam B, et al. Protection of rabbits against challenge with rabbit papillomaviruses by immunization with the $\mathrm{N}$ terminus of human papillomavirus type 16 minor capsid antigen L2. J Virol 2007; 81: 11585-11592. DOI: 10.1128/JVI.01577-07

63. Schiller JT, Lowy DR. Understanding and learning from the success of prophylactic human papillomavirus vaccines. Nat Rev Microbiol 2012; 10: 681-692. DOI: 10.1038/nrmicro2872

64. Einstein $\mathrm{MH}$, Baron $\mathrm{M}$, Levin $\mathrm{MJ}$, et al. Comparison of the immunogenicity of the human papillomavirus (HPV)-16/18 vaccine and the HPV-6/11/16/18 vaccine for oncogenic non-vaccine types HPV-31 and HPV-45 in healthy women aged 18-45 years. Hum Vaccin 2011; 7: 1359-1373. DOI: 10.4161/hv.7.12.18282

65. Einstein $\mathrm{MH}$, Baron $\mathrm{M}$, Levin $\mathrm{MJ}$, et al. Comparative immunogenicity and safety of human 
papillomavirus (HPV)-16/18 vaccine and HPV-6/11/16/18 vaccine: follow-up from months $12-24$ in a Phase III randomized study of healthy women aged 18-45 years. Hum Vaccin 2011; 7: 1343-1358. DOI: 10.4161/hv.7.12.18281

66. Einstein $\mathrm{MH}$, Levin MJ, Chatterjee $\mathrm{A}$, et al. Comparative humoral and cellular immunogenicity and safety of human papillomavirus (HPV)-16/18 AS04-adjuvanted vaccine and HPV-6/11/16/18 vaccine in healthy women aged 18-45 years: follow-up through Month 48 in a Phase III randomized study. Hum Vaccin Immunother 2014; 10: 3455-3465. DOI: 10.4161/hv.36117

67. Einstein $\mathrm{MH}$, Takacs $\mathrm{P}$, Chatterjee $\mathrm{A}$, et al. Comparison of long-term immunogenicity and safety of human papillomavirus (HPV)-16/18 AS04-adjuvanted vaccine and HPV-6/11/16/18 vaccine in healthy women aged 18-45 years: end-of-study analysis of a Phase III randomized trial. Hum Vaccin Immunother 2014; 10: 3435-3445. DOI: 10.4161/hv.36121

68. Hu YM, Huang SJ, Chu K, et al. Safety of an Escherichia coli-expressed bivalent human papillomavirus (types 16 and 18) L1 virus-like particle vaccine: an open-label phase I clinical trial. Hum Vaccin Immunother 2014; 10: 469-475. DOI: 10.4161/hv.26846

69. Zhao H, Lin ZJ, Huang SJ, et al. Correlation between ELISA and pseudovirion-based neutralisation assay for detecting antibodies against human papillomavirus acquired by natural infection or by vaccination. Hum Vaccin Immunother 2014; 10: 740-746.

70. Wu T, Hu YM, Li J, et al. Immunogenicity and safety of an E. coli-produced bivalent human papillomavirus (type 16 and 18) vaccine: A randomized controlled phase 2 clinical trial. Vaccine 2015; 33: 3940-3946. DOI: 10.1016/j.vaccine.2015.06.052

71. Dias D, Van Doren J, Schlottmann S, et al. Optimization and validation of a multiplexed luminex assay to quantify antibodies to neutralizing epitopes on human papillomaviruses $6,11,16$, and 18 . Clin Diagn Lab Immunol 2005; 12: 959-969. DOI: 10.1128/CDLI.12.8.959-969.2005

72. Opalka D, Lachman CE, MacMullen SA, et al. Simultaneous quantitation of antibodies to neutralizing epitopes on virus-like particles for human papillomavirus types $6,11,16$, and 18 by a multiplexed luminex assay. Clin Diagn Lab Immunol 2003; 10: 108-115.

73. Krajden M, Cook D, Yu A, et al. Human papillomavirus 16 (HPV 16) and HPV 18 antibody responses measured by pseudovirus neutralization and competitive Luminex assays in a two- versus three-dose HPV vaccine trial. Clin Vaccine Immunol 2011; 18: 418-423. DOI: 10.1128/CVI.00489-10

74. Opalka D, Matys K, Bojczuk $P$, et al. Multiplexed serologic assay for nine anogenital human papillomavirus types. Clin Vaccine Immunol 2010; 17: 818-827. DOI: 10.1128/CVI.00348-09

75. Brown MJ, Seitz H, Towne V, Muller M, Finnefrock AC. Development of neutralizing monoclonal antibodies for oncogenic human papillomavirus types $31,33,45,52$, and 58. Clin Vaccine Immunol 2014; 21: 587-593. DOI: 10.1128/CVI.00773-13

76. Group FIIS, Dillner J, Kjaer SK, et al. Four year efficacy of prophylactic human papillomavirus quadrivalent vaccine against low grade cervical, vulvar, and vaginal intraepithelial neoplasia and anogenital warts: randomised controlled trial. BMJ 2010; 341: c3493. DOI: 10.1136/bmj.c3493

77. Paavonen J, Naud P, Salmeron J, et al. Efficacy of human papillomavirus (HPV)-16/18 AS04-adjuvanted vaccine against cervical infection and precancer caused by oncogenic HPV types (PATRICIA): final analysis of a double-blind, randomised study in young women. Lancet 2009; 374 : 301-314. DOI: 10.1016/S0140-6736(09)61248-4

78. Herrero $\mathrm{R}$, Wacholder $\mathrm{S}$, Rodriguez $\mathrm{AC}$, et al. Prevention of persistent human papillomavirus infection by an HPV16/18 vaccine: a community-based randomized clinical trial in Guanacaste, Costa Rica. Cancer Discov 2011; 1: 408-419. DOI: 10.1158/2159-8290.CD-11-0131 
79. Romanowski B. Long term protection against cervical infection with the human papillomavirus: review of currently available vaccines. Hum Vaccin 2011; 7: 161-169.

80. Schiller JT, Castellsague X, Garland SM. A review of clinical trials of human papillomavirus prophylactic vaccines. Vaccine 2012; 30 Suppl 5: F123-138. DOI: 10.1016/j.vaccine.2012.04.108

81. Roteli-Martins CM, Naud P, De Borba $P$, et al. Sustained immunogenicity and efficacy of the HPV-16/18 AS04-adjuvanted vaccine: up to 8.4 years of follow-up. Hum Vaccin Immunother 2012; 8: 390-397. DOI: 10.4161/hv.18865

82. Shank-Retzlaff M, Wang F, Morley $\mathrm{T}$, et al. Correlation between mouse potency and in vitro relative potency for human papillomavirus Type 16 virus-like particles and Gardasil vaccine samples. Hum Vaccin 2005; 1: 191-197.

83. Serrano B, Alemany L, Tous S, et al. Potential impact of a nine-valent vaccine in human papillomavirus related cervical disease. Infect Agent Cancer 2012; 7: 38. DOI: 10.1186/1750-9378-7-38 84. Joura $E A$, Giuliano $A R$, Iversen $O E$, et al. A 9-valent $H P V$ vaccine against infection and intraepithelial neoplasia in women. N Engl J Med 2015; 372: 711-723. DOI: 10.1056/NEJMoa1405044 85. Petrosky E, Bocchini JA, Jr., Hariri S, et al. Use of 9-valent human papillomavirus (HPV) vaccine: updated HPV vaccination recommendations of the advisory committee on immunization practices. MMWR Morb Mortal Wkly Rep 2015; 64: 300-304.

86. Richards RM, Lowy DR, Schiller JT, Day PM. Cleavage of the papillomavirus minor capsid protein, L2, at a furin consensus site is necessary for infection. Proc Natl Acad Sci U S A 2006; 103: 1522-1527. DOI: $10.1073 /$ pnas.0508815103

87. Kondo K, Ishii Y, Ochi H, Matsumoto T, Yoshikawa H, Kanda T. Neutralization of HPV16, 18, 31, and 58 pseudovirions with antisera induced by immunizing rabbits with synthetic peptides representing segments of the HPV16 minor capsid protein L2 surface region. Virology 2007; 358: 266-272. DOI: 10.1016/j.virol.2006.08.037

88. Slupetzky K, Gambhira R, Culp TD, et al. A papillomavirus-like particle (VLP) vaccine displaying HPV16 L2 epitopes induces cross-neutralizing antibodies to HPV11. Vaccine 2007; 25: 2001-2010. DOI: 10.1016/j.vaccine.2006.11.049

89. Schellenbacher C, Kwak K, Fink D, et al. Efficacy of RG1-VLP vaccination against infections with genital and cutaneous human papillomaviruses. J Invest Dermatol 2013; 133: 2706-2713. DOI: 10.1038/jid.2013.253

90. Chen X, Liu H, Zhang $T$, et al. A vaccine of L2 epitope repeats fused with a modified IgG1 Fc induced cross-neutralizing antibodies and protective immunity against divergent human papillomavirus types. PLoS One 2014; 9: e95448. DOI: 10.1371/journal.pone.0095448

91. Seitz H, Dantheny T, Burkart F, Ottonello S, Muller M. Influence of oxidation and multimerization on the immunogenicity of a thioredoxin- 12 prophylactic papillomavirus vaccine. Clin Vaccine Immunol 2013; 20: 1061-1069. DOI: 10.1128/CVI.00195-13

92. Buck CB, Cheng $N$, Thompson CD, et al. Arrangement of $L 2$ within the papillomavirus capsid. J Virol 2008; 82: 5190-5197. DOI: 10.1128/JVI.02726-07 


\section{Figure Legends}

Fig. 1 The different forms, natural virion, pseudovirion, and VLP of papillomavirus and their applications. (A) Surface representation of the BPV cryoEM image reconstruction. The native virions were used in biological studies on viral assembly and cell entry, among others. (Adapted from [56]) (B) CryoEM image reconstructions of HPV16 pseudovirions that were used to develop the PsV-based neutralization assay. (Adapted from [92]) (C) CryoEM image reconstructions of HPV16 VLPs. Two VLP-based prophylactic vaccines have been globally approved for clinical use.

(Adapted from [57]) Native virion and pseudovirion are composed of the major L1 and the minor L2 proteins, They also both encapsidate DNA. However, VLPs do not contain the L2 protein, which is self-assembled from L1 protein only without DNA involvement. The structures of all three forms have been determined and they all showed icosahedral symmetry. Although the native virion and pseudovirion contain the L2 protein the precise structure of L2 is poorly resolved in all the available structures

Fig. 2 Three binding modes for neutralizing antibodies against papillomavirus capsid. (Left) mAb H11.B2 binding to HPV11 VLP (the DE loops on L1) indicates that the binding sites are located at the center of the capsomere. (Middle) mAb 5B6 binding to the two L1 molecules of the adjacent hexavalent capsomeres. (Right) mAb \#9 binding to the outer surface of the hexavalent capsomere. mAb H16.V5 binding to HPV16 VLP (FG and HI loops on L1) shows that H16.V5 only preferentially binds to the

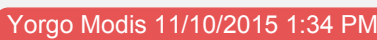

Deleted: Adopted

\section{Yorgo Modis 11/10/2015 1:34 PM}

Deleted: Adopted

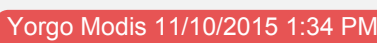

Deleted: Adopted

Yorgo Modis 11/10/2015 1:31 PM

Deleted: ,

Yorgo Modis 11/10/2015 1:31 PM

Deleted: In addition, $\mathrm{t}$

Yorgo Modis 11/10/2015 1:31 PM

Deleted: all

Yorgo Modis 11/10/2015 1:31 PM

Deleted: form

Yorgo Modis 11/10/2015 1:31 PM

Deleted: es

Yorgo Modis 11/10/2015 1:32 PM

Deleted: a single

Yorgo Modis 11/10/2015 1:32 PM

Deleted: spatial

Yorgo Modis 11/10/2015 1:32 PM

Deleted: demonstrated

Yorgo Modis 11/10/2015 1:32 PM

Deleted: an

Yorgo Modis 11/10/2015 1:33 PM

Deleted: a

Yorgo Modis 11/10/2015 1:33 PM

Deleted: .

Yorgo Modis 11/10/2015 1:33 PM

Deleted: To date,

Yorgo Modis 11/10/2015 1:33 PM

Deleted: still unavailable 
1012

1013

1014

1015

1016

1017

1018

1019

1020

1021

1022

1023

1024 1025

1026

1027

1028

1029

1030

1031 outer surface of the hexavalent capsomeres. The inset pictures were used to further clarify the interaction models between antibody and antigen.

Fig. 3 HPV16.V5 binding sites and specific amino acid residues involved at the

Ab-Ag interface. (A) The footprint of antibodies H16.V5, H16.1A H16.14J and

H263.A2 mapped to the stereographic projection of a capsomer. The virus surface

was represented as a quilt format for the jcosahedral symmetric unit, with the polar

angles $\varphi$ and $\theta$ representing the latitude and Jongitude. The color bar indicates the

different antigenic L1 loops and each mAb epitope was denoted using dashed circle.

The common epitope indicated by arrow includes all residues identified in each $\mathrm{mAb}$

footprint. (Adapted from [61]) (B) A 3D reconstruction structure based on cryo-EM

data of mAb H16.V5 binding to HPV16 VLP (FG and HI loops on L1) shows that

H16.V5 only binds to the hexavalent capsomeres but not the pentavalent capsomeres.

(EMDB:28370). (Adapted from [57]) (C) The 3D structural model of mAb H16.V5

binding to HPV16 VLP, with magenta for H16.V5 binding site on FG loop and red on

HI loop, and cyan for VLP. (Adapted from [30]) (D) The HPV16.V5 Fab binds 17

residues across five loops from two neighboring L1 proteins. The contacting residues

are shown as spheres with the residue names and loops labeled. Within the five loops,

three loops, BC, DE, FG, are contributed by the first L1 protein. The other two loops,

$\mathrm{DE}$ and $\mathrm{HI}$ are contributed by the second neighboring L1 protein. (Adapted from [59])

(E) The accurate epitope location of HPV16.V5 on the exterior flexible loops with a

total of identified 16 amino acid residues. The loops with red represent the major
Yorgo Modis 11/10/2015 1:30 PM

Deleted: elucidate

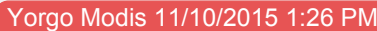

Deleted: antibodides

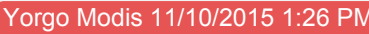

Deleted: icosahedrala

Yorgo Modis 11/10/2015 1:26 PM

Deleted: longitude.The

Yorgo Modis 11/10/2015 1:35 PM

Deleted: Adopted

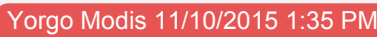

Deleted: Adopted

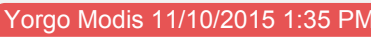

Deleted: Adopted

Yorgo Modis 11/10/2015 1:34 PM

Deleted: Adopted 

a second neighboring L1. 
CRPV, Belnap et al, JMB, 1996

HPV-1 \& BPV-1, Baker et al, Biophys. J, 1991

A

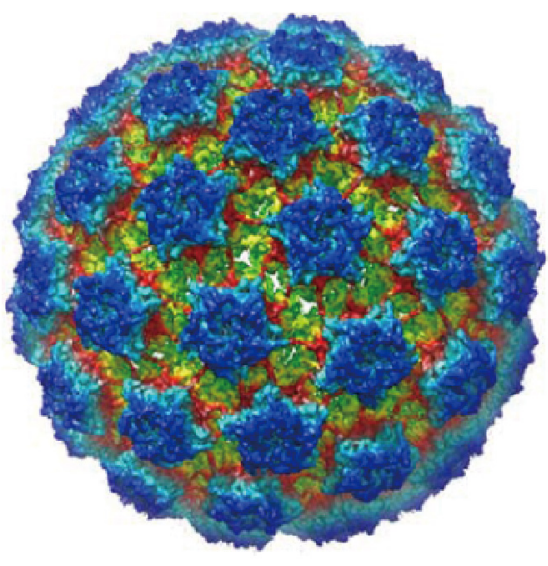

Biological studies including viral assembly, cell entry and

HPV16, Bunk et al, JV, 2008

HPV16, Lee et al, JVI, 2015

B

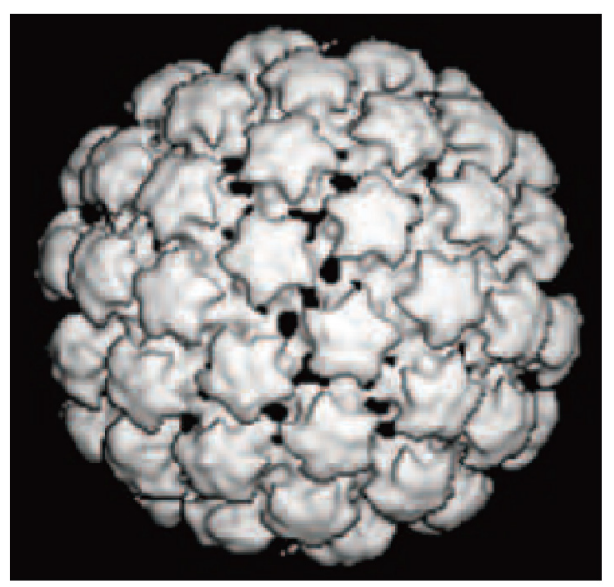

$+$

Neutralization assay
HPV11/16, Zhao et al, HVI, 2014

C

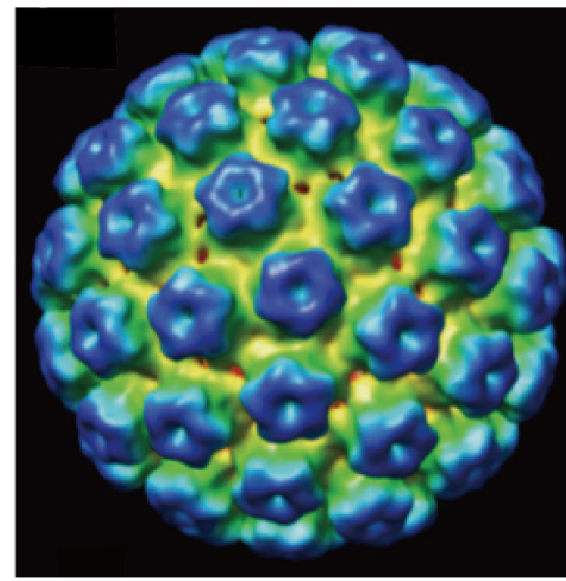

DNA others

Application

$+$

Fig. 1 


\section{$\pi$
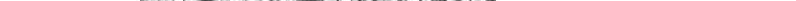
A

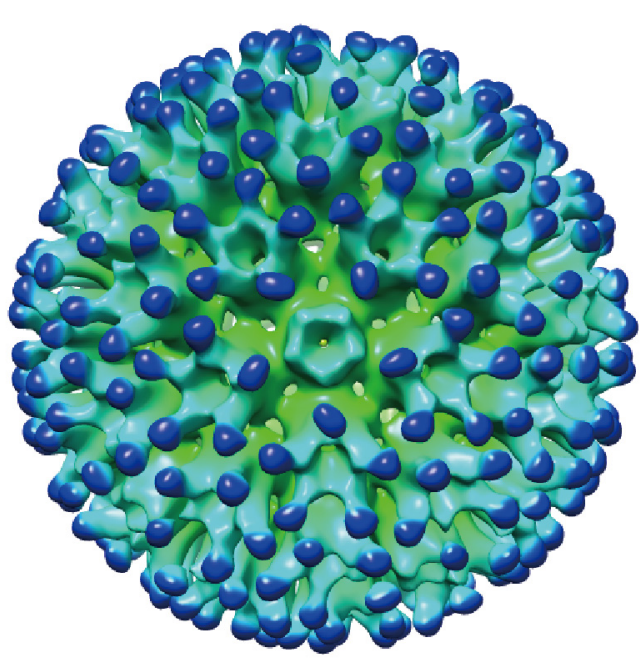

B

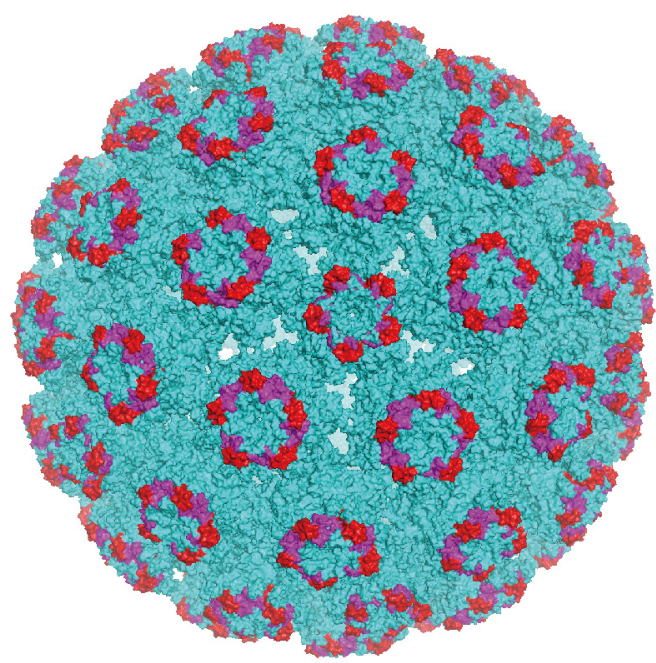

C

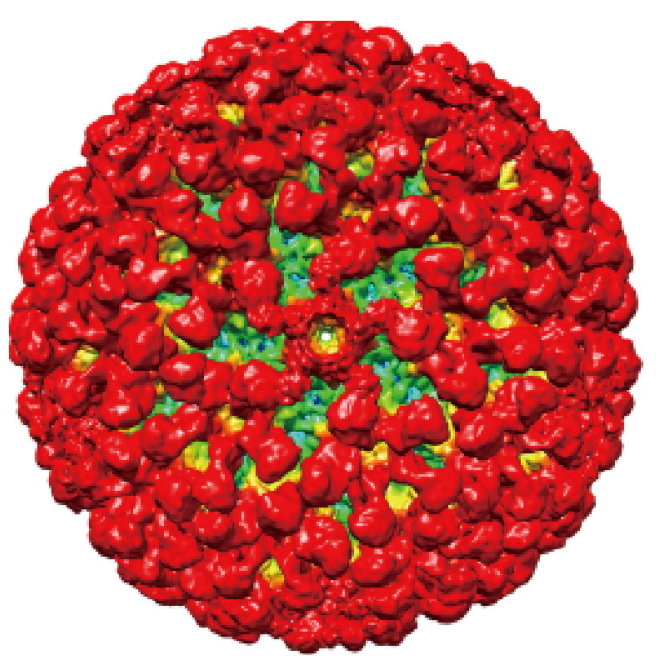

D

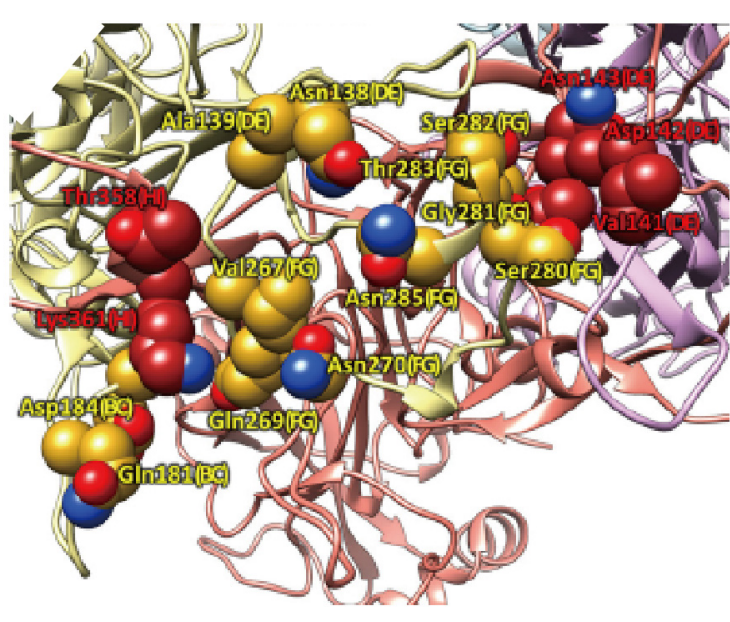

Fig. 3 